\title{
A numerical analysis of the elastic-plastic peel test
}

\author{
H. Hadavinia ${ }^{1}$, L. Kawashita, A.J. Kinloch ${ }^{2}$, D.R. Moore and J.G. Williams
}

Department of Mechanical Engineering, Imperial College London, Exhibition Rd, London, SW7 2AZ, UK.

( ${ }^{1}$ Present address: Kingston University, Kingston on Thames, Surrey)

( ${ }^{2}$ Corresponding author)

\begin{abstract}
.
The adhesive fracture energy, $G_{c}$, is determined from two types of elastic-plastic peel tests (i.e. the single-arm $90^{\circ}$ and T-peel methods) and a linear-elastic fracture-mechanics (LEFM) test method (i.e. the tapered double-cantilever beam, TDCB method). A rubbertoughened epoxy adhesive, with both aluminium-alloy and steel substrates, has been used in the present work to manufacture the bonded joints. The peel tests are then modelled using numerical methods. The overall approach to modelling the elastic-plastic peel tests is to employ a finite-element analysis (FEA) approach and to model the crack advance through the adhesive layer via a node-release technique, based upon attaining a critical plastic strain in the element immediately ahead of the crack tip. It is shown that this 'critical plastic strain fracture model (CPSFM)' results in predicted values of the steady-state peel loads which are in excellent agreement with the experimentally-measured values. Also, the resulting values of $G_{c}$, as determined using the FEA CPSFM approach, have been found to be in excellent agreement with values from previously-reported analytical and direct-measurement methods. Further, it has been found that the calculated values of $G_{c}$ are independent of whether a standard LEFM test or an elastic-plastic peel test method is employed. Therefore, it has been demonstrated that the value of the adhesive fracture energy, $G_{c}$, is independent of the geometric parameters studied and the value of $G_{c}$ is indeed a characteristic of the joint, in this
\end{abstract}


case for cohesive fracture through the adhesive layer. Finally, it is noted that the FEA CPSFM approach promises considerable potential for the analysis of peel tests which involve very extensive plastic deformation of the peeling arm and for analysing, and predicting, the performance of more complex adhesively-bonded geometries which involve extensive plastic deformation of the substrates.

Keywords: adhesives, finite-element analysis, fracture energies, joints, modelling, peel tests.

\section{Nomenclature}

$\begin{array}{ll}a & \text { crack length } \\ b & \text { width } \\ G & \text { overall (input) peel energy } \\ G_{c} & \text { adhesive fracture energy } \\ G_{p} & \text { plastic component of the energy in the peel arm(s) } \\ h & \text { height } \\ m & \text { geometry factor } \\ n & \text { work-hardening exponent } \\ P & \text { load } \\ W_{A} & \text { thermodynamic work of adhesion } \\ \overline{\varepsilon_{p c}} & \text { critical uniaxial tensile plastic strain } \\ \mathcal{E}_{p}^{*} & \text { multiaxial equivalent plastic strain } \\ \mathcal{E}_{p c}^{*} & \text { critical multiaxial equivalent plastic strain } \\ \sigma_{e} & \text { equivalent (von Mises) stress } \\ \sigma_{H} & \text { hydrostatic stress } \\ \theta & \text { peel angle }\end{array}$




\section{Introduction}

The value of the adhesive fracture energy, $G_{c}$, should be characteristic of the joint and, ideally, independent of geometric parameters [1]. Nevertheless, it is recognised that, since the value of $G_{c}$ includes plastic and viscoelastic energy dissipation which occurs locally at the crack tip, it will be a function of the rate and temperature at which the peel test is conducted. (Only if such energy losses are reduced to virtually zero, and the locus of joint failure is exactly along the bimaterial interface, will the value of $G_{c}$ be equivalent to the thermodynamic work of adhesion, $W_{A}[2]$.) The adhesive fracture energy, $G_{c}$, of adhesive joints may be readily ascertained from linear-elastic fracture-mechanics (LEFM) methods, and indeed a British Standard (BS7991-2001) now exists [3] to measure the LEFM Mode I value, largely as a result of the efforts of the European Structural Integrity Society (ESIS) TC4 Committee as described by Blackman et al. [4,5]. Notwithstanding, the LEFM test specimens are relatively complex and expensive to make and test, and many industries would far prefer to deduce the value of $G_{c}$ from the common and widely used 'peel test', as shown schematically in Figs. 1 and 2.

The determination of the adhesive fracture energy, $G_{c}$, from the total external energy required to generate peel fracture, $G$, with a plastically-deforming peel-arm is a classical problem in fracture analysis. It involves the determination of the plastic component of the energy, $G_{p}$, in the arm(s) which is subtracted from $G$. As such, it is a somewhat rare example of elastic-plastic fracture where such a segregation of energies can be made. In some cases $G_{p}$ is up to $85 \%$ of the total $G$. There are experimental schemes for finding $G_{p}$ using rollers to control the curvature of the peel arms as in a mandrel test [6,7] or by measuring the curvature directly [8]. Generally, however, $G_{p}$ is found via some form of analysis to determine the curvature and then $G_{p}$ computed via an elastic-plastic bending solution. An analytical version of such a solution is available [9-11] which employs large displacement beam analysis and corrects for local deformations around the debond front. However, such methods are limited, in that much detail is lost in the assumptions made and there are considerable attractions in performing a numerical analysis.

The formulation of such an numerical analysis presents substantial challenges, since large deformations are involved. In particular the initiation phase during which the steadystate, from which the partitioning is made, is established is challenging. However, finiteelement analysis (FEA) codes such as 'ABAQUS' can, in principle, cope with such problems and the steady-state solutions can be found. Whilst global partitioning of energy is possible, 
it is more usual to determine $G_{c}$ via local node-release at the debond point or via local stress and displacement contours, since this is computationally efficient. If an experimental steadystate load is known, then the solution for $G_{c}$ must be assumed and the load computed, and matching the experimental load requires considerable iteration. Such methods are very inefficient and require long computing times [12].

A popular method for overcoming this problem is to use a cohesive zone model for the debonding process in which $G_{c}$ is prescribed [10, 13-19]. In addition, however, a traction stress must also now be used and there is no way of predetermining what this should be. Judicious choices can give satisfactory results and reasonable efficiency but the stress is not a material property and varies with geometry $[10,16]$. The present paper explores an alternative scheme in which a strain criterion is prescribed for the determination of $G_{c}$. Since the strains are computed everywhere it is easy to prescribe some value, say in the crack tip element, and then determine $G_{c}$ in the usual way and this leads to very efficient computation. There is, however, a problem in prescribing a critical strain in stress fields of different constraint factors. This is addressed here by using a modified form of the Rice-Tracey criterion [20] in which the critical strain used is a function of the hydrostatic stress. Such a criterion implies some form of cavitation failure process, which indeed occurs in polymeric adhesives which are rubber-toughened.

\section{Experimental procedures}

\subsection{Materials}

The adhesive chosen was 'Permabond ESP110', a single-part, hot-cured epoxy-paste adhesive. It consists of a rubber-toughened epoxy resin filled with a high fraction of aluminium powder (up to $30 \%$ weight) for improved mechanical properties at elevated temperatures. The surface treatment employed for all substrates (i.e. the aluminium alloy and mild steel materials) was grit-blasting using alumina grit (mesh-size: 400) followed by immersion in a degreasing bath of $1,1,1$ trichloroethylene $\left(10 \mathrm{~min}\right.$ at $\left.60{ }^{\circ} \mathrm{C}\right)$. The aluminiumalloy substrates were then further treated in a chromic-acid solution (30 min at $63{ }^{0} \mathrm{C}$ ) according to the recommendations of BS 7773 [21]. This was followed by immersing the substrates in a bath of circulating water $(20 \mathrm{~min})$, followed by a final rinse with distilled water. The substrates were then dried in a hot-air oven $\left(1 \mathrm{~h}\right.$ at $\left.60{ }^{\circ} \mathrm{C}\right)$ and kept in a desiccator for approximately $24 \mathrm{~h}$ prior to bonding. During joint assembly, steel wires were used as spacers to control the thickness of the adhesive layer, and poly(tetrafluoroethylene) films served as pre-cracks. The test specimens were clamped in dedicated rigs which held the joints 
in place during the curing process. Cure of the adhesive layer took place in a hot-air oven for $45 \mathrm{~min}$ at $150{ }^{\circ} \mathrm{C}$. The final adhesive layer thickness was approximately $0.4 \mathrm{~mm}$ for all the test specimens.

\subsection{Peel tests}

The adhesive fracture energy, $G_{c}$, was measured using both single-arm $90^{\circ}$ peel and Tpeel tests; where the test configurations are shown schematically in Figs. 1 and 2, respectively. Two different peel arm materials were employed: an aluminium alloy designation 5754-O and a mild steel. Both materials were supplied in the form of sheets with thickness of about $1 \mathrm{~mm}$. The peel arms consisted of rectangular strips $220 \mathrm{~mm} \times 20 \mathrm{~mm} \times 1$ $\mathrm{mm}$, guillotined from large sheets in parallel orientation $\left(0^{0}\right)$ to the rolling direction. For the single-arm peel joints, aluminium-alloy sheets of $180 \mathrm{~mm} \times 20 \mathrm{~mm} \times 10 \mathrm{~mm}$ served as the rigid base plates. The dimensions of the peel arms were measured prior to joint preparation. Single-arm peel tests, using an applied peel angle of $90^{\circ}$, and T-peel tests were conducted, as mentioned above. All peel tests were performed at the same temperature, humidity $\left(21{ }^{\circ} \mathrm{C}, 55\right.$ $\% \mathrm{RH})$ and crack growth rate $(5 \mathrm{~mm} / \mathrm{min})$. In all cases, only the steady-state peel forces were considered.

Single-arm $90^{\circ}$ peel test specimens had their base plates bolted onto a linear bearing, which was then mounted on the testing machine. The free extremity of the peel arm was bent around a $10 \mathrm{~mm}$ radius roller so it could be easily clamped by the upper grip to give an applied peel angle of $90^{\circ}$. Peeling proceeded with a crosshead speed of $5 \mathrm{~mm} / \mathrm{min}$. Three replicate specimens were tested and, again, only steady-state forces were considered.

T-peel specimens were connected to the 'Instron' testing machine with the use of end blocks (a) to allow free rotation of the peel arms and (b) to minimize the transient behaviour at the start of the test. A crosshead speed of $10 \mathrm{~mm} / \mathrm{min}$ resulted in a crack growth of $5 \mathrm{~mm} / \mathrm{min}$. Note that all tests were 'asymmetric' with respect to the manner in which the crack propagated through the adhesive layer, i.e. the crack propagated closer to one of the substrates during steady-state peeling, see Fig. 3. The consequent mismatch in stiffness between the two peel arms then resulted in the bonded-end specimen being inclined at different angle to that of $90^{\circ}$ to the horizontal. The angle formed between the bonded-end of the specimen and the loading axis was measured via the analysis of digital photographs taken during the tests. Three replicate specimens were tested. 


\subsection{Uniaxial tensile tests}

In order to ascertain the values of the power-law modelling parameters, see below, the materials used for the peel arm were tested in uniaxial tension using an 'Instron' universal testing machine equipped with a video extensometer to measure the axial strain. The dogbone shaped specimens were machined from the same grade of sheets, and followed the same orientation ( $0^{0}$ to the rolling direction), as used for the peel arms. The geometry of the tensile specimens was based on BS EN 10002 [22] and had with a parallel gauge-length of $57 \mathrm{~mm}$, a width of $12.5 \mathrm{~mm}$ and a thickness of $1.0 \mathrm{~mm}$. The gauge length as measured by the extensometer was $50 \mathrm{~mm}$. Tests were conducted at room temperature $\left(21^{0} \mathrm{C}\right)$ with a crosshead speed of $1 \mathrm{~mm} / \mathrm{min}$, resulting in a strain rate of approximately $3 \times 10^{-4} \mathrm{~s}^{-1}$.

\subsection{TDCB tests}

The adhesive fracture energy, $G_{c}$, of the adhesive was directly measured using a LEFM tapered double-cantilever beam (TDCB) test, as shown schematically in Fig. 4. The substrates were machined, using a computer-controlled milling machine from 2014A aluminium-alloy sheets. The height, $h$, of a beam was selected such that the change in compliance with crack length, $a$, was constant; and this was achieved by ensuring that the geometry factor term, $m$, was constant. The final beams were $10 \mathrm{~mm}$ in width, $300 \mathrm{~mm}$ in length and had a constant geometry factor $m=2 \mathrm{~mm}^{-1}$ [3-5]. Where $m$, the geometry factor, is given by:

$$
m=\frac{3 a^{2}}{h^{3}}+\frac{1}{h}
$$

where $a$ is the crack length and $h$ is the corresponding height of the substrate beam.

The TDCB tests were performed according to BS 7991 [3] and the values of $G_{c}$ were also deduced as described in this standard method. A travelling optical microscope was used for recording the value of the crack length. Tests were conducted at standard conditions $\left(21^{\circ} \mathrm{C}, 55 \% \mathrm{RH}\right)$ for three replicate specimens. The crosshead speed was $0.1 \mathrm{~mm} / \mathrm{min}$, and crack length measurements were taken after approximately every $2 \mathrm{~mm}$ of propagation. No plastic deformation was observed in any of the TDCB joints, thus confirming that these tests followed the basic principles of linear-elastic fracture-mechanics. 


\section{The numerical approach}

\subsection{Introduction}

The overall approach to the numerical analysis that we have taken is to employ a finiteelement analysis (FEA) approach and model crack advance through the adhesive layer via a node-release technique. The nodes are released, and so the crack allowed to advance, when the multiaxial equivalent plastic strain, $\varepsilon_{p}^{*}$, in the element immediately ahead of the crack attains a critical value, $\varepsilon^{*}{ }_{p c}$. Thus, we have developed a 'critical plastic strain fracture model (CPSFM)'. However, the multiphase adhesive used in the present study undergoes plastic void initiation and growth in the plastic, or damage, zone ahead of the crack tip. Indeed, this is the major toughening micromechanism in such materials [23,24]. From the work of Rice and Tracey [20] it is well established that, when such a micromechanism occurs, the value of the $\varepsilon_{p c}^{*}$ is strongly influenced by the local stress-state and the value of the ratio $\varepsilon_{p c}^{*} / \overline{\varepsilon_{p c}}$ is related to the stress ratio, $\sigma_{H} / \sigma_{e}$, by the expression [25]:

$$
\boldsymbol{\varepsilon}_{p c}^{*} / \frac{0.521}{\boldsymbol{\varepsilon}_{p c}}=\frac{0}{\sinh \left(1.5 \sigma_{H} / \boldsymbol{\sigma}_{e}\right)}
$$

where $\varepsilon_{p c}^{*}$ is the critical multiaxial equivalent plastic strain, $\overline{\varepsilon_{p c}}$ is the critical uniaxial tensile plastic strain, $\sigma_{H}$ is the hydrostatic stress and $\sigma_{e}$ is the equivalent (von Mises) stress. This relationship is plotted in Fig. 5 . The value of the critical uniaxial tensile plastic strain, $\overline{\varepsilon_{p c}}$, is taken to be a constant and in the present work is ascertained by interrogating the LEFM TDCB, as described below. (Its value cannot readily be directly determined from uniaxial tensile tests conducted using the adhesive material, since such tests tend to show brittle fracture prior to ductile failure.)

For the TDCB tests, the aluminium-alloy substrates were modelled as linear-elastic materials with a modulus of 69GPa. For the peel tests, the aluminium-alloy peel arms were modelled as power-law materials [10] with modulus, uniaxial tensile yield-stress and workhardening exponent values of $66 \mathrm{GPa}, 85 \mathrm{MPa}$ and $\mathrm{n}=0.22$, respectively. The corresponding values for the steel peel-arm were $207 \mathrm{GPa}, 124 \mathrm{MPa}$ and $n=0.2$. The adhesive was modelled as an elastic-plastic material with modulus and uniaxial tensile yield-stress values of $4 \mathrm{GPa}$, and $35 \mathrm{MPa}$, and possessing a maximum tensile fracture stress of $70 \mathrm{MPa}$ at a strain of 0.045 . 


\subsection{The CPSFM approach}

The detailed steps used in the present work are:

i. The first step was to ascertain the value of the critical uniaxial tensile plastic strain, $\overline{\varepsilon_{p c}}$, which would then be kept constant in value in all the subsequent studies. This was achieved by undertaking a finite-element numerical analysis of the LEFM TDCB test. Various mesh sizes were examined and that selected, to give a good representation of the adhesive and to give convergence of the solution in a reasonable time-scale, is shown in Fig. 6(d). The value of the critical multiaxial equivalent plastic strain, $\varepsilon_{p c}^{*}$, for node release which gave the failure load, which was independently experimentallymeasured, was then ascertained for this mesh size. Next, knowing from the finiteelement analysis results the stress ratio, $\sigma_{H} / \sigma_{e}$, in the element ahead of the crack tip, the Rice-Tracey relationship (see Eq. (2)) was used to calculate the corresponding value of $\overline{\varepsilon_{p c}}$ that gave this value of $\varepsilon_{p c}^{*}$, and thus gave the correct value of the experimentallymeasured failure load.

ii. Having so determined the value of $\overline{\varepsilon_{p c}}$, this value of $\overline{\varepsilon_{p c}}$ was then kept constant for all subsequent studies of the peel tests, using the corresponding mesh size to that shown in Fig. 6(d) for the modelling of such joints.

iii. To then undertake a finite-element numerical analysis of the elastic-plastic peel tests:

- Firstly, to ascertain the value of the stress ratio, $\sigma_{H} / \sigma_{e}$, in the element ahead of the crack tip (i.e. the peel front).

- Secondly, to use this value of the stress ratio, $\sigma_{H} / \sigma_{e}$, together with the now fixed, constant, value of $\overline{\varepsilon_{p c}}$, ascertained as described in (i) above, to calculate the value of the critical multiaxial equivalent plastic strain, $\varepsilon_{p c}^{*}$, using the Rice-Tracey relationship, i.e. Eq.(2).

- Thirdly, to use this value of $\varepsilon_{p c}^{*}$ to determine when to release the node immediately ahead of the crack tip. This was achieved by applying an increasing displacement to the peel test arm(s) until, in the element immediately ahead of the crack tip, the condition $\varepsilon^{*}{ }_{p} \geq \varepsilon^{*}{ }_{p c}$ was met; where $\varepsilon^{*}$ is the multiaxial equivalent plastic strain at a given applied displacement.

- Fourthly, the predicted loads and displacements, as a function of the extent of theoretical crack growth, for a given peel test may be ascertained simply by applying the criterion release of $\varepsilon_{p}^{*} \geq \varepsilon_{p c}^{*}$ for successive nodes, and so allowing the crack to advance through the adhesive layer. 
- $\quad$ Finally, the predicted values of the adhesive fracture energy, $G_{c}$, of the adhesive joints were ascertained by using the J-integral method and/or the virtual crack closure method. For the former method, the number of contours employed was eight, and the results from the last three contours were averaged to give the value of $G_{c}$. There was always an excellent agreement between the two methods used to ascertain the value of $G_{c}$.

The FEA program used was 'ABAQUS', with 'PATRAN' being used for the mesh modelling.

\subsection{The LEFM tapered double-cantilever beam (TDCB) test}

As noted above, the LEFM TDCB test, using aluminium-alloy substrates, was employed to ascertain the value of the critical uniaxial tensile plastic strain, $\overline{\varepsilon_{p c}}$, which would then be kept constant in value for all subsequent analyses of the LEFM TDCB and elasticplastic peel tests. For the linear-elastic deforming substrate arms, four-noded plane-strain elements were used. In the case of the $0.4 \mathrm{~mm}$ thick elastic-plastic adhesive layer, four elements (CPE4R type) were placed across the layer and a cohesive crack path along the centre of the adhesive layer was assumed. The two faces of this crack path were connected together using multi-point constraint (MPC). The MPC from adjoining nodes was removed when the condition for crack growth to occur, i.e. when the condition $\varepsilon_{p}^{*} \geq \varepsilon_{p c}^{*}$, was satisfied. This process was implemented via a MPC-user subroutine. Displacement control was used throughout the modelling work.

Fig. 6 schematically shows the meshes used for the LEFM TDCB specimen; where Fig. 6 (a) is the overall representation and Figs. 6 (b) to (e) show the different element sizes used for the meshes which represented the adhesive layer: namely element sizes of 1.8, 0.6, $0.2,0.1 \mathrm{~mm}$, respectively. It was found that, as expected, the value of the critical multiaxial equivalent plastic strain, $\varepsilon_{p c}^{*}$, and hence the calculated value of the critical uniaxial tensile plastic strain, $\overline{\varepsilon_{p c}}$, needed to fit the experimentally-measured failure load was mesh dependent. Hence, a mesh size was selected, and then kept constant, for all the subsequent studies on modelling the different types of adhesive joint test-specimens.

It was found that the mesh with an element size of $0.2 \mathrm{~mm}$ gave a good representation for the adhesive layer with a reasonably short time needed for a given computation run. The 
number of elements employed in this FEA model was 23,196. The corresponding value of the critical multiaxial equivalent plastic strain, $\varepsilon_{p c}^{*}$, for node-release which yielded the measured failure load for the LEFM TDCB specimen was $1.32 \times 10^{-3}$. The resulting value of the critical uniaxial tensile plastic strain, $\overline{\varepsilon_{p c}}$, was calculated from the Rice and Tracey relationship to be 0.04. (The value of the stress ratio stress, $\sigma_{H} / \sigma_{e}$, in the element ahead of the crack tip, which is needed in this calculation was determined from the finite-element analysis.)

\subsection{The elastic-plastic peel tests}

For the T-peel tests, 13,538 four-noded plane-strain elements (CPE4R type) were used for each peel arm and the mesh size used was virtually identical to that for the LEFM TDCB tests. Thus, again, in the case of the $0.4 \mathrm{~mm}$ thick elastic-plastic adhesive layer four elements (CPE4R type) were placed across the layer and a crack path either (a) along the centre of the adhesive layer, or (b) very close to a peel arm/adhesive interface was assumed. In the latter case, the crack path was located $50 \mu \mathrm{m}$ from the peel arm, in accord with the experimental observations, see Fig. 3. The two faces of these crack paths were connected together using multi-point constraint (MPC). The MPC from adjoining nodes was removed when the condition for crack growth to occur, i.e. when the condition $\varepsilon^{*}{ }_{p} \geq \varepsilon^{*}{ }_{p c}$, was satisfied. The node-release process was implemented via a MPC-user subroutine. The corresponding value of $\varepsilon_{p c}^{*}$ for node-release was deduced as described above, using $\overline{\varepsilon_{p c}}=0.04$ and the Rice and Tracey relationship. (The value of the stress ratio, $\sigma_{H} / \sigma_{e}$, in the element ahead of the crack tip (i.e. the peel front) was determined from the finite-element analysis.) The value of $\varepsilon_{p c}^{*}$ so calculated was $2.08 \times 10^{-3}$ for both the aluminium-alloy and steel T-peel tests.

Modelling the crack path as being within the adhesive layer but being located only 50 $\mu \mathrm{m}$ from a peel arm/adhesive interface did indeed result in a very accurate prediction of the bonded-end of the T-peel specimen being inclined at different angle to that of $90^{\circ}$ to the horizontal, as schematically shown in Fig. 3. Namely, an angle of about $60^{\circ}$ for the value of $\theta_{B}$ in Fig. 3 was experimentally measured and predicted. However, interestingly, the FEA CPSFM results demonstrated that modelling the crack path either (a) along the centre of the adhesive layer, or (b) within the adhesive layer but being located $50 \mu \mathrm{m}$ from a peel arm/adhesive interface, had no significant effect on the predicted peeling load per unit width, or the associated predicted value of the adhesive fracture energy, $G_{c}$. In the discussion below the values corresponding to the crack path within the adhesive layer, but being located $50 \mu \mathrm{m}$ from the peel arm/adhesive interface, have been used; since this corresponds to the experimental observations. 
For the single-arm $90^{\circ}$ peel test specimens, the crack path was again modelled as being within the adhesive layer but being located $50 \mu \mathrm{m}$ from the aluminium-alloy peel arm/adhesive interface, in agreement with the experimental observations. The mesh size used was again virtually identical to that for the LEFM TDCB tests; and again the MPC from adjoining nodes was removed when the condition for crack growth to occur, i.e. when the condition $\varepsilon_{p}^{*} \geq \varepsilon_{p c}^{*}$, was satisfied. The value of ${\varepsilon^{*}}_{p c}$, deduced, from using $\overline{\varepsilon_{p c}}=0.04$ and the Rice and Tracey relationship, was $9.79 \times 10^{-3}$. (The value of the stress ratio stress ratio, $\sigma_{H} / \sigma_{e}$, in the element ahead of the crack tip (i.e. the peel front) was again determined from the finiteelement analysis.)

Finally, it should be noted, that mesh-sizes for the peel tests exactly equivalent to the LEFM TDCB test were, as might be expected, difficult to achieve. However, an examination of the mesh sensitivity of the results indicated that the errors in the values of the failures load and adhesive fracture energies predicted for the peel tests, due to the very minor variations in the mesh sizes used for the peel tests compared to that employed for the LEFM TDCB test, would only be in error by about $\pm 1 \%$.

\section{Results and discussion}

\subsection{The LEFM tapered double-cantilever beam (TDCB)test}

As described above, the LEFM TDCB test, using aluminium-alloy substrates, was employed to ascertain the value of the critical uniaxial tensile plastic strain, $\overline{\varepsilon_{p c}}$, which was then kept constant in value for all subsequent analyses of the elastic-plastic peel tests. The method outlined in Sections 3.2 and 3.3 described above was followed and the results are shown in Table 1. As may be seen, a value of $\overline{\varepsilon_{p c}}$ of 0.04 gives a value for the load per unit width, $P / b$, for steady-state crack growth which is in excellent agreement with the experimentally measured value, as indeed would be expected. Further, the resulting value of $G_{c}$ from the modelling studies is also in agreement with the value deuced from the analytical approach given in BS7991 [3]. This latter observation clearly gives confidence in the CPSFM approach. Thus, for all subsequent studies, the critical uniaxial tensile plastic strain, $\overline{\varepsilon_{p c}}$, was maintained at a value of 0.04 with the mesh size also being held essentially constant, as noted above. 


\subsection{The elastic-plastic peel tests}

The values for the steady-state load for the $90^{\circ}$ peel and T-peel tests are shown in Table 2, from both the experiments and as predicted from using the FEA CPSFM, as described above. As may be seen, the agreement is excellent for (a) both types of peel test and (b) when either the aluminium-alloy or steel substrates are employed.

\subsection{Values of the adhesive fracture energy, $G_{c}$}

The values of the adhesive fracture energy, $G_{c}$, are shown in Table 3. There are several noteworthy points.

Firstly, the value of $G_{c}$, as analytically determined [3] from the LEFM TDCB tests, is independent of the type of substrate, as would be expected. Also, since the measured loads from the TDCB tests, using aluminium-alloy substrates, were used to fix the value of $\overline{\varepsilon_{p c}}$ at a value of 0.04 , then the agreement between the values of $G_{c}$ from the experimental measurements and the FEA CPSFM approach would be expected to be very good for this particular LEFM test specimen, as is indeed the case.

Secondly, for the various types of peel test, the values of $G_{c}$ as determined using the analytical method [9-11] and the experimental mandrel method [6,7] are given, and good agreement is seen between all these different tests and various methods for ascertaining values of $G_{c}$. Thirdly, it should be noted that the analytical method [9-11] could not be readily employed for the T-peel tests using the steel peel-arm, since the extent of plastic bending of the steel arm was so extensive that it invalidated this analytical-model approach. (For example, if the analytical route is employed to obtain a value of $G_{c}$ for the T-peel test using the steel peel-arm, then a very high value of some $2200 \mathrm{~J} / \mathrm{m}^{2}$ is deduced, presumably due to the very high correction [26] that is necessary for the extensive plasticity that occurs in the steel peel arms.)

Fourthly, for the various types of peel test, the values of $G_{c}$, as determined using the FEA CPSFM approach, are also stated. It may be clearly seen that the $G_{c}$ from this new FEA node-release approach are in excellent agreement with the values from all the other previously-reported methods. Further, the FEA CPSFM is also capable of yielding a sensible value of $G_{c}$ from the $90^{\circ}$ peel test employing the steel peel-arm. 
Finally, however, it should be noted that the $G_{c}$ value for the $90^{\circ}$ peel tests from the FEA CPSFM approach is about $840 \mathrm{~J} / \mathrm{m}^{2}$, which is somewhat lower than that deduced from the other methods. It should be further noted that the degrees of constraint, as defined by the stress ratio, $\sigma_{H} / \sigma_{e}$, are almost the same in value for both the TDCB and T-peel test specimens, but that these values are relatively high compared to that for the $90^{\circ}$ peel test; i.e. values of about two compared to unity (see Tables 1 and 2). Thus, it appears that the lower degree of constraint for the $90^{\circ}$ peel test, as defined by the stress ratio, $\sigma_{H} / \sigma_{e}$, certainly does not lead to an increase in the value of $G_{c}$. This is an interesting observation which warrants further investigation. It may arise from either (a) the local degree of constraint being relieved by the voiding which occurs ahead of the crack tip in the adhesive layer, and/or (b) the degree of constraint always being relatively high in the adhesive layer. Such that, in either case, the value of $G_{c}$ has become independent of the value of the stress ratio, $\sigma_{H} / \sigma_{e}$.

To summarise, from Table 3, it may be concluded that the calculated values of the adhesive fracture energy, $G_{c}$, are independent of the geometric parameters studied and the value of $G_{c}$ is indeed a characteristic of the joint, in this case for cohesive fracture through the adhesive layer. Thus, the present work leads to the conclusion that the value of $G_{c}$ is independent of whether a standard LEFM test or an elastic-plastic peel test method is employed.

\section{Conclusions}

The overall approach to the numerical analysis of the elastic-plastic peel tests that we have taken is to employ a finite-element analysis (FEA) approach and model crack advance through the adhesive layer via a node-release technique based upon attaining a critical plastic strain in the element immediately ahead of the crack tip. It has been shown that this new 'critical plastic strain fracture model (CPSFM)' results in values of the steady-state peel loads which are in excellent agreement with the experimentally measured values. Further, the resulting values of the adhesive fracture energy, $G_{c}$, are in excellent agreement with those ascertained from previously-reported analytical and mandrel-method techniques. Also, it may be concluded that the values of $G_{c}$ are independent of the geometric parameters studied. Indeed, the value of $G_{c}$ is a characteristic of the joint, in this case for cohesive fracture through the adhesive layer. Thus, the present work confirms the hypothesis [1] that the value of $G_{c}$ is independent of whether a standard LEFM test or an elastic-plastic peel test is undertaken. Finally, the FEA CPSFM approach promises considerable potential for the analysis of peel tests which involve very extensive plastic deformation of the peel arm and for 
analysing, and predicting, the performance of more complex adhesively-bonded geometries which involve extensive plastic deformation of the substrates.

\section{Acknowledgements}

The authors would wish to thank the EPSRC, UK for financial support through a Platform Grant Award for H. Hadavinia, the Royal Academy of Engineering, UK for support for D.R. Moore and IMRE, Singapore for support for L.F. Kawashita. 


\section{References}

[1] Kinloch AJ. Adhesion and adhesives: science and technology. London \& New York. Chapman and Hall; 1987.

[2] Andrews EH and Kinloch AJ. Mechanics of adhesive failure. Proc Royal Society 1973;A332:385-400.

[3] BSI. Determination of the mode I adhesive fracture energy, $\mathrm{G}_{\mathrm{IC}}$, of structural adhesives using the double cantilever beam (DCB) and tapered double-cantilever beam (TDCB) specimens. 2001:BS 7991.

[4] Blackman BRK, Kinloch AJ, Paraschi M and Teo WS. Measuring the mode I adhesive fracture energy, $G_{I C}$, of structural adhesive joints: The results of an international roundrobin. Int J Adhes and Adhes 2003;23:293-305.

[5] Blackman BRK and Kinloch AJ. in 'Facture mechanics testing methods for polymers, adhesives and composites’ edited by D.R. Moore, A. Pavan and J.G. Williams; Elsevier Science, Amsterdam, 2001:225-270.

[6] Breslauer E and Trocynski TJ. Experimental determination of the peel adhesion strength for metallic foils. Adhes Sci Technol 1998;12;367-382.

[7] Kawashita LF, Moore DR and Williams JG. The development of a mandrel peel test for the measurement of adhesive fracture toughness of epoxy-metal laminates. J. Adhes 2004;80:147-167.

[8] Kawashita LF, Moore DR and Williams JG. The influence of peel arm curvature in the determination of adhesive fracture toughness of metal-polymer laminates. J Materials Sci 2005;40:4541-4548.

[9] Kinloch AJ, Lau CC and Williams JG. The peeling of flexible laminates. Int. J. Fract 1994;66:45-70.

[10] Georgiou I, Hadavinia H, Ivankovic I, Kinloch AJ, Tropsa V and Williams JG. Cohesive zone models and the plastically deforming peel test. J Adhes 2003;79:239265.

[11] Imperial College London Website, Peel test protocols: 'ICPeel' http:/www.me.ic.ac.uk/AACgroup/

[12] Blackman BRK, Kinloch AJ, Taylor, AC and Wang Y. The impact wedge-peel performance of structural adhesives. J Materials Sci 2000;35:1867-1884. 
[13] Tvergaard V and Hutchinson JW. On the toughness of ductile adhesive joints. J Mech Phys Solids 1996;44:789-800.

[14] Wei Y and Hutchinson JW. Interface strength, work of adhesion and plasticity in the peel test. Int J Fract 1998;93:315-333.

[15] Chowdhury SR and Narasimham R. A finite element analysis of quasi-static crack growth in a pressure-sensitive constrained ductile layer. Eng Fract Mechs 2000;66:551557.

[16] Blackman BRK, Hadavinia H, Kinloch AJ and Williams JG. The use of cohesive zone models to study the fracture of fibre composites and adhesively-bonded joints. Int $\mathrm{J}$ Fract 2003;119:25-46.

[17] Ferracin T, Landis CM, Delannay F and Pardoen T. On the determination of the cohesive zone properties of an adhesive layer from the analysis of the wedge-peel test. Int J Solids Struct 2004;40:2889-2904.

[18] Li S, Thouless MD, Waas AM, Schroeder JA and Zavattieri PD. Use of mode-I cohesive-zone models to describe the fracture of an adhesively-bonded polymer-matrix composite. Comp Sci Tech 2005;65:281-293.

[19] Pardoen T, Ferracin T, Landis CM, and Delannay F. Constraint effects in adhesive joint fracture. J Mech Phys Solids 2005;53:1951-1983.

[20] Rice JR and Tracey DM. On the ductile enlargement of voids in triaxial stress fields. J Mech Phys Solids 1969;17:201-217.

[21] BSI. Code of practice for cleaning and preparation of metal surfaces. 1995:BS 7773.

[22] BSI. Tensile testing of metallic materials. Method of test at ambient temperatures. 2001:BS EN 10002-1.

[23] Kinloch AJ, Shaw SJ, Tod DA and Hunston DL. Deformation and fracture behaviour of a rubber-toughened epoxy. I - microstructure and fracture studies. Polymer 1983;24:1341-1355.

[24] Kinloch AJ. Toughening epoxy adhesives to meet today's challenges. Materials Research Society Bulletin 2003;28:445-449.

[25] Yatomi M, Bettinson AD, O'Dowd, NP and Nikbin KM. Modelling of damage and failure in notched bar multiaxial creep tests. Fatigue Fract Eng Mater Struct 2004;27:283-295. 
[26] Kinloch AJ and Williams JG. Comments on "Determining the toughness of plastically deforming joints”. J Materials Sci Letters 1998;17:813-814. 
Table 1. Comparison of the measured and predicted failure loads per unit width, $P / b$, and adhesive fracture energies, $G_{c}$, from the FEA CPSFM calculations and the experiments, respectively, for the TDCB LEFM test using aluminium alloy. (The FEA CPSFM was fitted using a value of $\overline{\varepsilon_{p c}}$ of 0.04 in the Rice and Tracey relationship.)

\begin{tabular}{|c|c|c|c|}
\hline Analysis Method & $\begin{array}{c}\text { Triaxiality Ratio, } \\
\sigma_{H} / \sigma_{\boldsymbol{e}}\end{array}$ & $\begin{array}{c}\text { Load per unit } \\
\text { width, } \mathbf{P} / \boldsymbol{b} \\
\mathbf{( N / \mathbf { m m } )}\end{array}$ & $\begin{array}{c}\boldsymbol{G}_{\boldsymbol{c}} \\
\left(\mathbf{J} / \mathbf{m}^{2}\right)\end{array}$ \\
\hline FEA model & 2.3 & 86 & 1070 \\
\hline $\begin{array}{c}\text { Experimental } \\
\text { (BS7991:2001) }\end{array}$ & Not applicable & $85 \pm 4$ & $1080 \pm 100$ \\
\hline
\end{tabular}


Table 2. Comparison of the measured and predicted peel loads per unit width, $P / b$, from the FEA CPSFM calculations and the experiments, respectively. (The FEA CPSFM was undertaken using a value of $\bar{\varepsilon}_{p c}$ of 0.04 in the Rice and Tracey relationship.)

\begin{tabular}{|c|c|c|c|c|}
\hline Test & Substrate & Method & $\begin{array}{l}\text { Triaxiality Ratio, } \\
\qquad \sigma_{H} / \sigma_{e}\end{array}$ & $\begin{array}{c}\text { Load per unit } \\
\text { width, }(P / b) \\
(\mathrm{N} / \mathrm{mm})\end{array}$ \\
\hline $90^{\circ}$ Peel & Aluminium alloy & FEA CPSFM & 1.0 & 6.4 \\
\hline $90^{\circ}$ Peel & Aluminium alloy & Experimental & Not applicable & $6.1 \pm 0.1$ \\
\hline T-peel & Aluminium alloy & FEA CPSFM & 2.0 & 3.9 \\
\hline T-peel & Aluminium alloy & Experimental & Not applicable & $3.4 \pm 0.1$ \\
\hline T-peel & Steel & FEA CPSFM & 2.0 & 6.6 \\
\hline T-peel & Steel & Experimental & Not applicable & $6.7 \pm 0.2$ \\
\hline
\end{tabular}


Table 3. Comparison of values of the adhesive fracture energies, $G_{c}$, from the various test methods and analysis routes. (The FEA CPSFM was undertaken using a value of $\overline{\varepsilon_{p c}}$ of 0.04 in the Rice and Tracey relationship.)

\begin{tabular}{|c|c|c|c|}
\hline Test & Substrate & Analysis Method & $\begin{array}{c}\boldsymbol{G}_{\mathbf{c}} \\
\mathbf{( J / \mathbf { m } ^ { 2 } )}\end{array}$ \\
\hline TDCB & Aluminium alloy & FEA CPSFM & 1070 \\
\hline TDCB & Aluminium alloy & Analytical (LEFM) & $1080 \pm 100$ \\
\hline TDCB & Steel & Analytical (LEFM) & $1020 \pm 60$ \\
\hline $90^{\circ}$ Peel & Aluminium alloy & FEA CPSFM & 840 \\
\hline $90^{\circ}$ Peel & Aluminium alloy & Analytical & $1080 \pm 50$ \\
\hline $90^{\circ}$ Peel & Aluminium alloy & Mandrel test & $1190 \pm 150$ \\
\hline T-peel & Aluminium alloy & FEA CPSFM & 1100 \\
\hline T-peel & Aluminium alloy & Analytical & $1050 \pm 20$ \\
\hline T-peel & Steel & FEA CPSFM & 995 \\
\hline
\end{tabular}




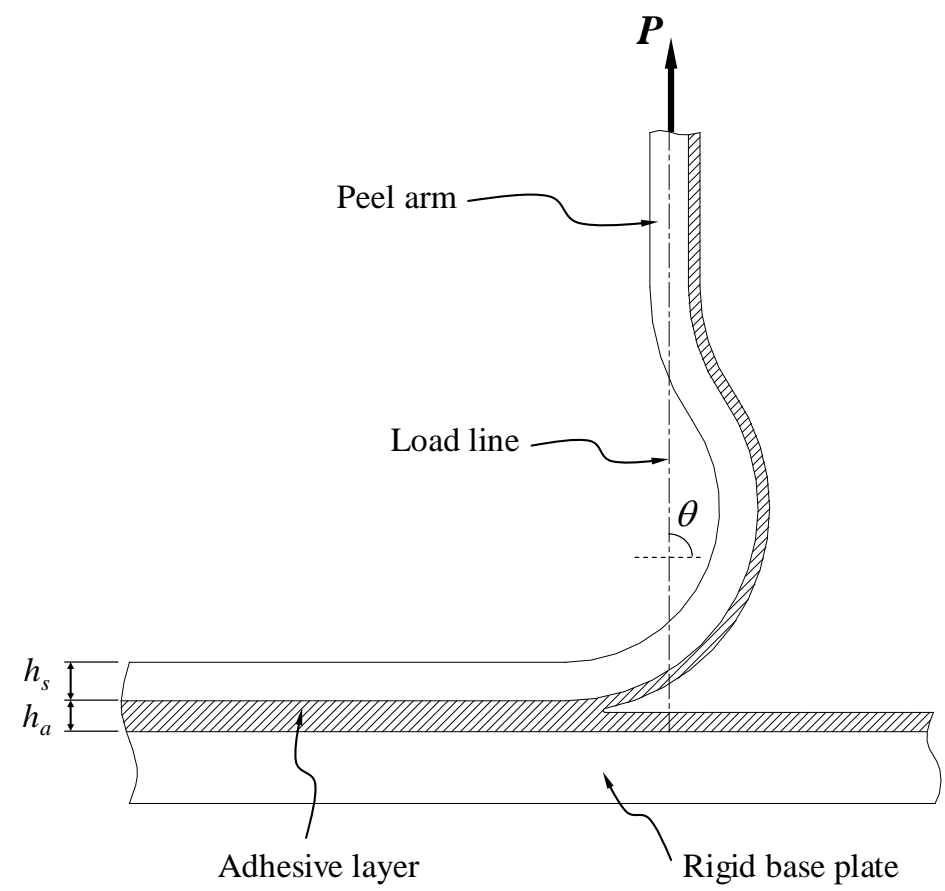

Fig. 1. The single-arm $90^{\circ}$ peel test. 


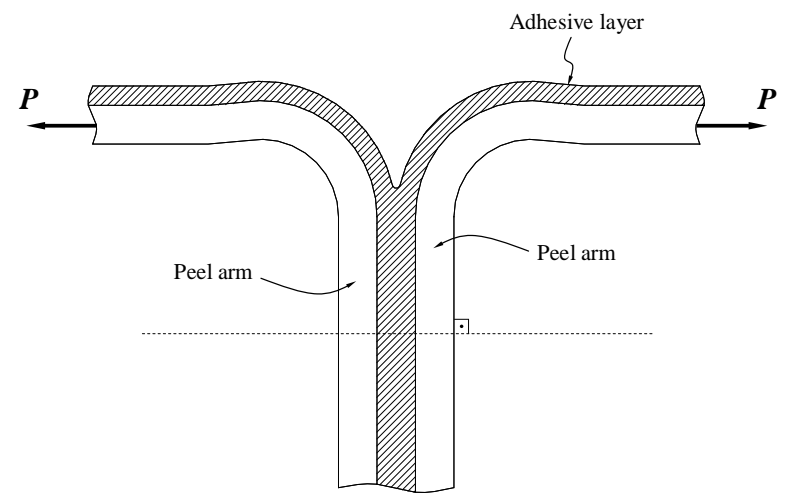

Fig. 2. The T-peel test. 


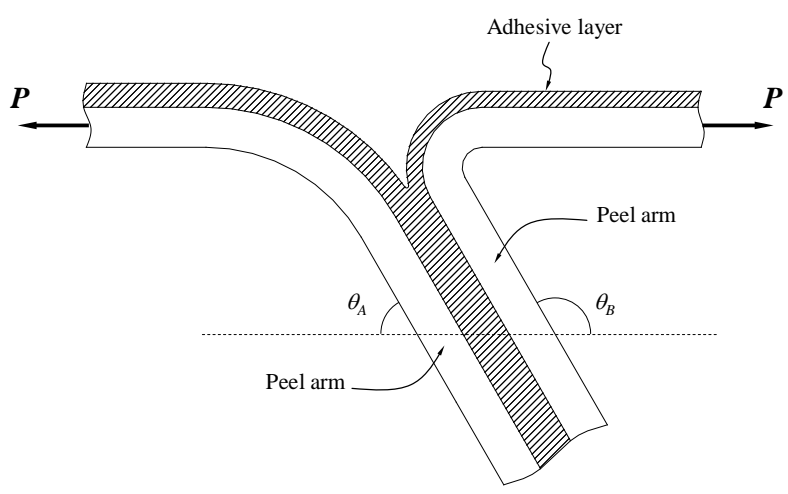

Fig. 3. The T-peel test with the crack path being within the adhesive layer (total thickness $0.4 \mathrm{~mm}$ ) but being located only $50 \mu \mathrm{m}$ from a peel arm/adhesive interface. 


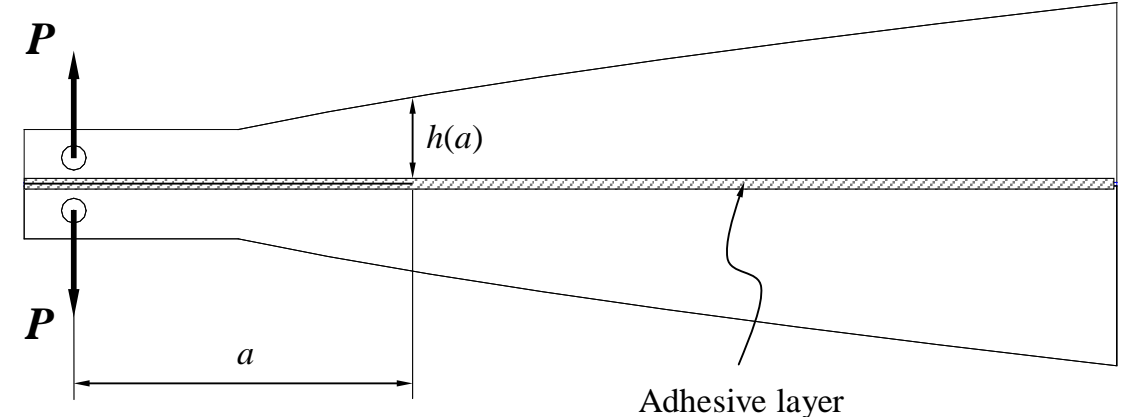

Adhesive layer

Fig. 4. The LEFM tapered double-cantilever beam (TDCB) test. 


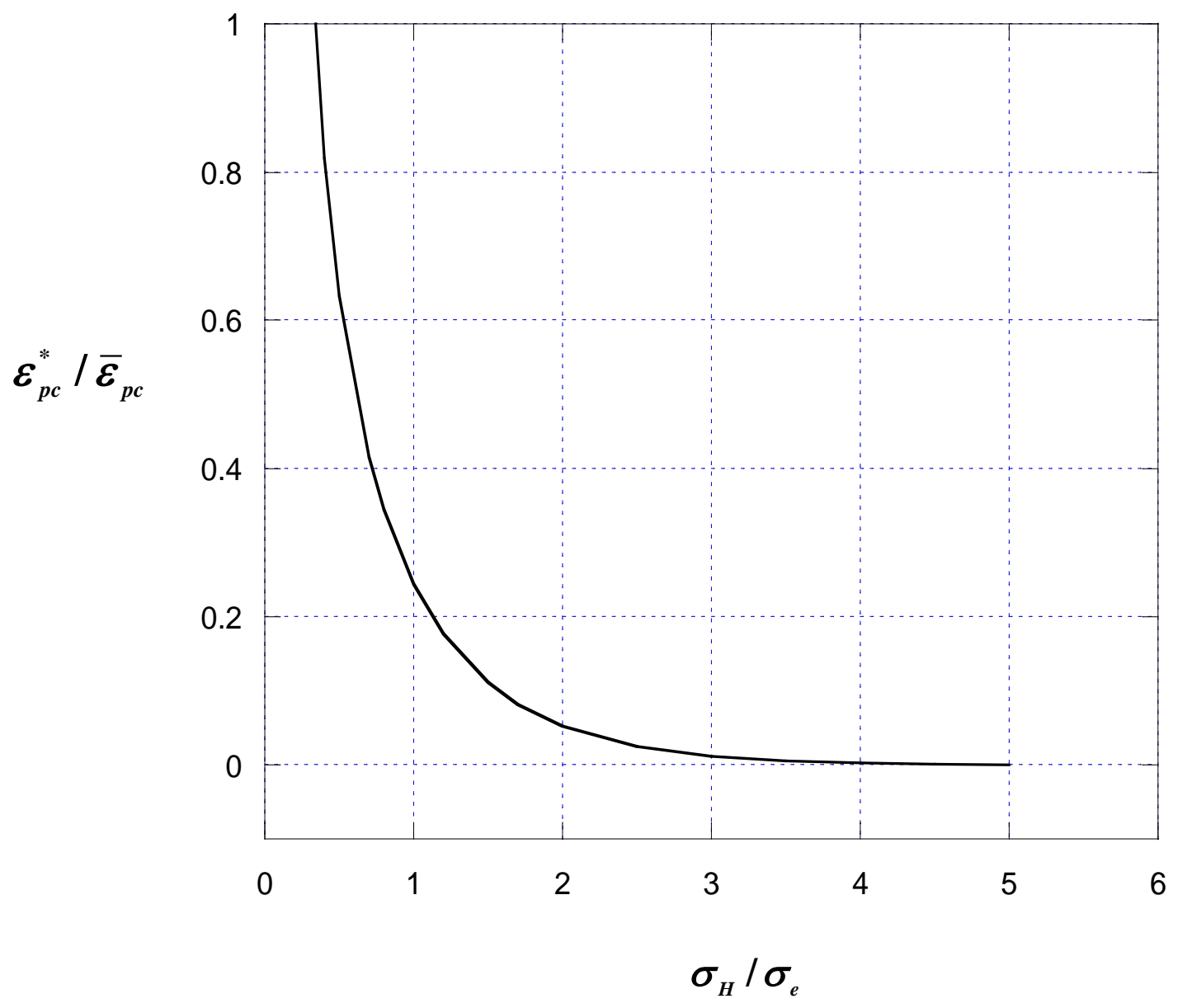

Fig. 5. The Rice-Tracey relationship. 


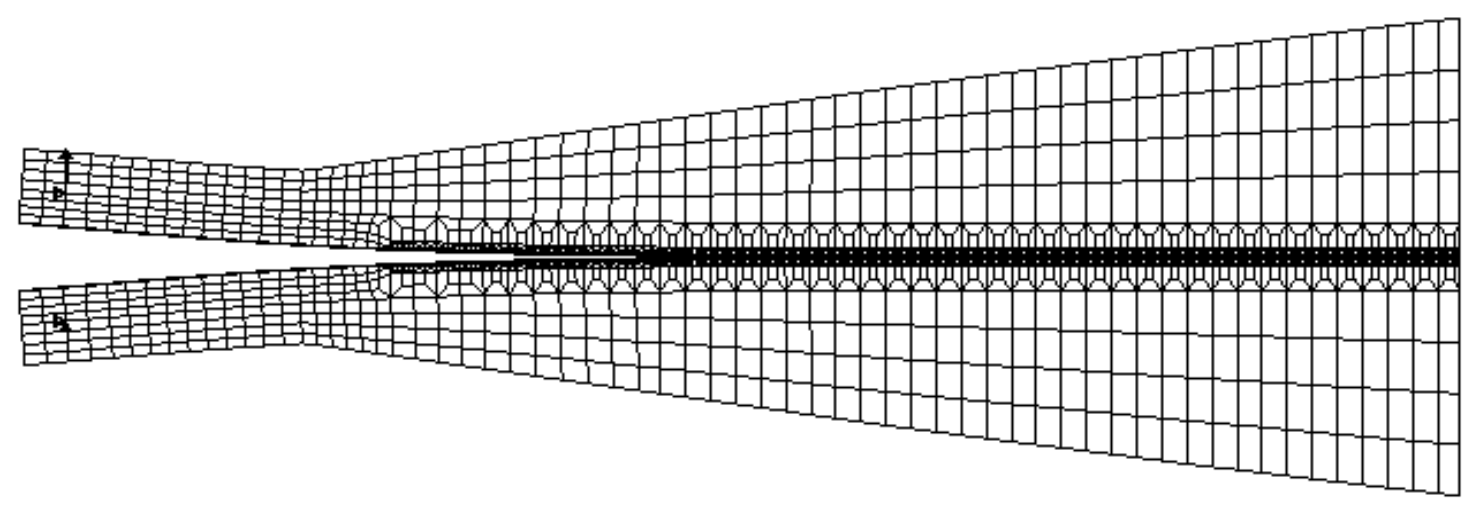

(a)

(b)

(c)
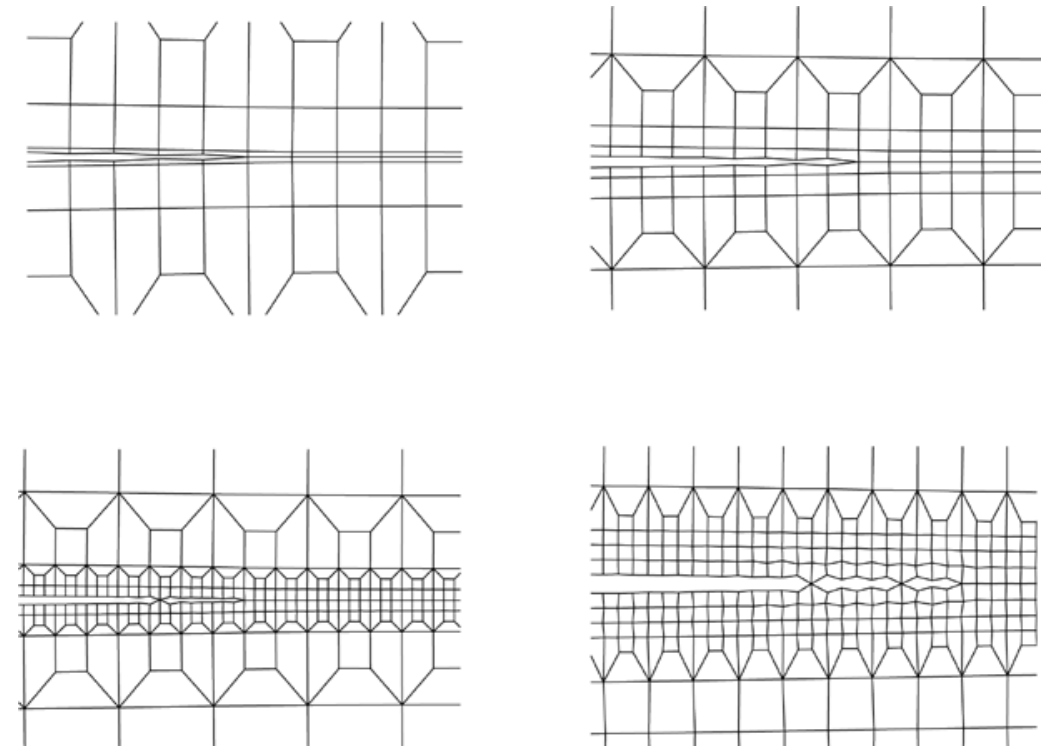

(d)

(e)

Fig. 6. Schematic of the meshes used for the LEFM TDCB specimen. (a) is the overall representation; and (b) to (e) represent the different elements sizes used for the meshes representing the adhesive layer: element sizes of $1.8,0.6,0.2,0.1 \mathrm{~mm}$, respectively. 